\title{
新型咖啡酸五环三萜酯的合成与保肝活性
}

\author{
贾 畑*, $a$ 孙学华 $b$ 邵腾飞 ${ }^{a}$ 金树根 ${ }^{b}$ 高月求 ${ }^{b}$ 王建武 $*, a$ \\ $\left({ }^{a}\right.$ 山东大学化学与化工学院 济南 250100) \\ $\left({ }^{b}\right.$ 上海中医药大学附属曙光医院 上海 201210)
}

\begin{abstract}
摘要 以天然的咖啡酸、甘草次酸和齐墩果酸为原料, 采用侧基保护、还原、酰化与去保护等步骤, 将咖啡酰基及氢 化咖啡酰基引入到甘草次酸和齐墩果酸的 3-位差基上，以较高收率合成了 4 个新的咖啡酸及氢化咖啡酸五环三萜酯类 化合物，通过 IR, ${ }^{1} \mathrm{H}$ NMR, HRMS 等方法确定了化合物的结构.
\end{abstract}

\section{Synthesis of Novel Caffeyl Triterpenic Esters and Their Hepatic Protective Effect}

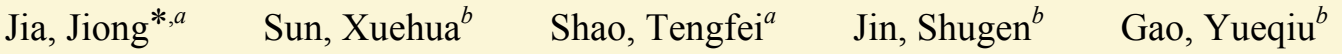 \\ Wang, Jianwu ${ }^{*, a}$ \\ ( ${ }^{a}$ School of Chemistry and Chemical Engineering, Shandong University, Jinan 250100) \\ ( ${ }^{b}$ Shanghai Shuguang Hospital, Shanghai University of Traditional Chinese Medicine, Shanghai 201210)
}

\begin{abstract}
Four new caffeyl triterpenic esters were synthesized from natural caffeic acid, glycyrrhetinic acid and oleanolic acid. The structures of all products were characterized by IR, ${ }^{1} \mathrm{H}$ NMR and HRMS techniques. The activities of the compounds to protect liver was tested and compound $9 \mathbf{c}$ showed good hepatic protective effect.

Keywords caffeyl glycyrrhetinate; caffeyl oleanolic ester; synthesis; hepatic protection
\end{abstract}

甘草次酸具有抗炎 ${ }^{[1]}$ 、保肝 ${ }^{[2]}$ 、抗溃疡 ${ }^{[3]}$ 、抗肿瘤、 抗艾滋病毒等功效 ${ }^{[4]}$, 本课题组曾对甘草次酸衍生物的 合成与活性做过一些研究工作 ${ }^{[5]}$, 并发现了具有较好活 性的新化合物. 齐墩果酸与甘草次酸有相似结构, 具有 保肝 ${ }^{[6]}$ 、抗炎 ${ }^{[7]}$ 等生物活性. 咖啡酸是一种天然的双着基 苯丙烯酸, 广泛存在于多种植物中, 具有抗菌抗病毒、 抗氧化等多种生物活性 ${ }^{[8]}$. 咖啡酸齐墩果酸酯在植物成 份中已被发现，并具有抗肿瘤等生物活性 ${ }^{[9,10]}$.

由于甘草次酸与齐墩果酸均具有一定的保肝活性, 为了探索咖啡酸酰化的五环三萜酯的合成及其生物活 性, 寻找高效低毒的新型保肝药物分子, 我们采用拼合 法, 将咖啡酰基及氢化咖啡酰基引入到甘草次酸和齐墩 果酸的 3-位差基上, 设计了 4 个新型咖啡酸及氢化咖啡 酸五环三萜酯类化合物, 并采用侧基保护、酰化与去保 护等步骤, 完成了化合物的合成, 用红外、核磁及质谱 等方法, 确定了它们的化学结构, 并初步测定了它们的
保肝活性. 结果发现, 引入咖啡酰基及氢化咖啡酰基的 五环三萜酯改变了其本身的生物活性, 氢化咖啡酸甘草 次酸酯 9c 表现出较好的保肝活性. 该结果为进一步开 发新的保肝药物建立了基础. 化合物 9 的合成路线见 Scheme 1 .

\section{1 实验部分}

\section{1 试剂与仪器}

X-4 数字显示显微熔点测定仪(北京泰克仪器有限 公司生产), 熔点未加校正; Bruker AV DPX300 核磁共 振仪, TMS 为内标; Shimadzu FTIR28400S 红外光谱仪; Q-TOF6510 质谱仪(Agilent); 甘草次酸(GA)、齐墩果酸 $(\mathrm{OA}) 、$ 咖啡酸均为 $98 \%$.纯度, 购自山东曙光植物制品有 限公司; 柱层析硅胶(200 300 目)购自烟台海洋化工 厂; 其他试剂均为市售分析纯; 所用的无水溶剂均经过 严格处理, 在使用前蒸出.

*E-mail: jwwang@sdu.edu.cn; jiongjia@sdu.edu.cn

Received August 29, 2011; revised November 3, 2011; accepted December 31, 2011. 


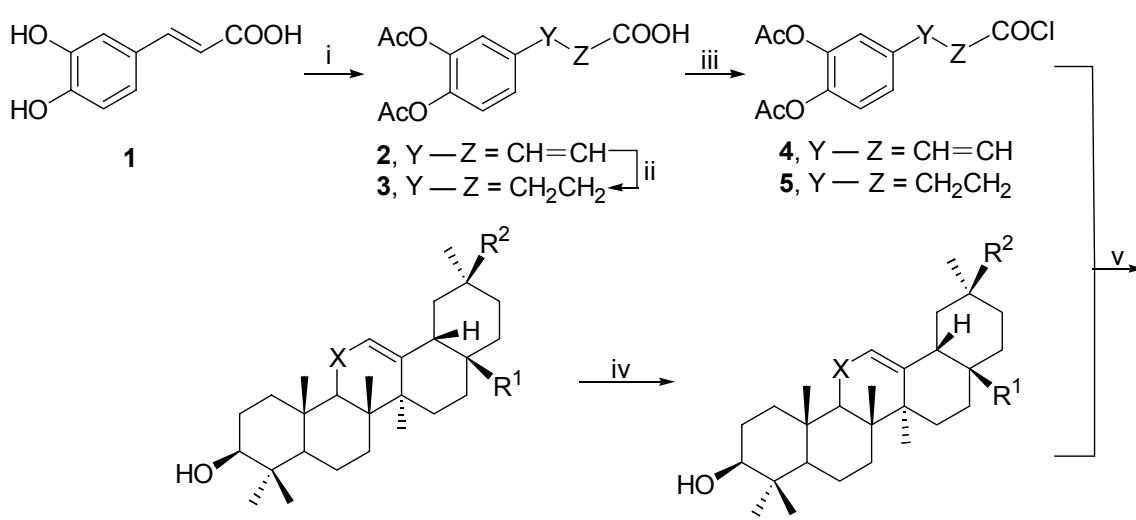

$\mathrm{GA}: \mathrm{R}^{1}=\mathrm{CH}_{3}, \mathrm{R}^{2}=\mathrm{CO}_{2} \mathrm{H}, \mathrm{X}=\mathrm{C}=\mathrm{O}$

6, $\mathrm{R}^{1}=\mathrm{CH}_{3}, \mathrm{R}^{2}=\mathrm{CO}_{2} \mathrm{CH}_{3}, \mathrm{X}=\mathrm{C}=\mathrm{O}$

$O A: \mathrm{R}^{1}=\mathrm{CO}_{2} \mathrm{H}, \mathrm{R}^{2}=\mathrm{CH}_{3}, \mathrm{X}=\mathrm{CH}_{2}$

7, $\mathrm{R}^{1}=\mathrm{CO}_{2} \mathrm{Et}, \mathrm{R}^{2}=\mathrm{CH}_{3}, \mathrm{X}=\mathrm{CH}_{2}$

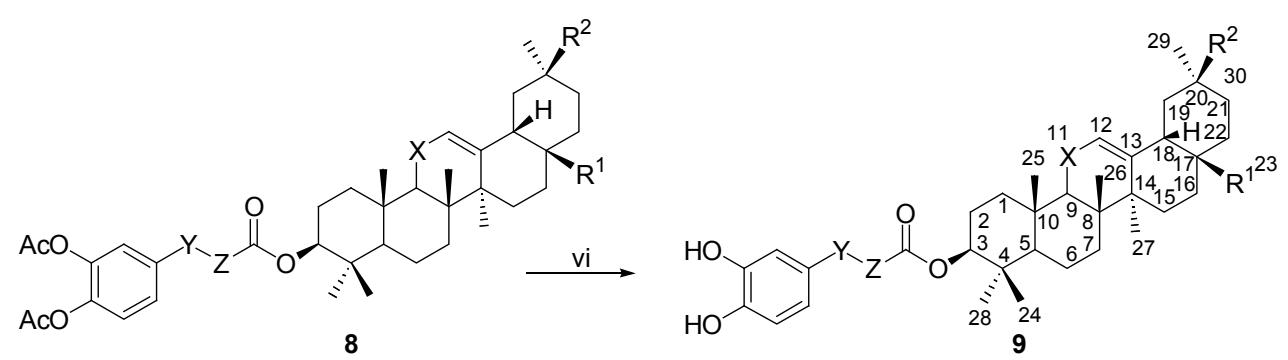

8a and 9a, $\mathrm{R}^{1}=\mathrm{CH}_{3}, \mathrm{R}^{2}=\mathrm{CO}_{2} \mathrm{CH}_{3}, \mathrm{X}=\mathrm{C}=\mathrm{O}, \mathrm{Y}-\mathrm{Z}=\mathrm{HC}=\mathrm{CH}$; $\mathbf{8 b}$ and $\mathbf{9 b}, \mathrm{R}^{1}=\mathrm{CO}_{2} \mathrm{Et}, \mathrm{R}^{2}=\mathrm{CH}_{3}, \mathrm{X}=\mathrm{CH}_{2}, \mathrm{Y}-\mathrm{Z}=\mathrm{HC}=\mathrm{CH}$ 8c and 9c, $R^{1}=\mathrm{CH}_{3}, \mathrm{R}^{2}=\mathrm{CO}_{2} \mathrm{CH}_{3}, \mathrm{X}=\mathrm{C}=\mathrm{O}, \mathrm{Y}-\mathrm{Z}=\mathrm{CH}_{2} \mathrm{CH}_{2} ; \mathbf{8 d}$ and $9 \mathrm{~d}, \mathrm{R}^{1}=\mathrm{CO}_{2} \mathrm{Et}, \mathrm{R}^{2}=\mathrm{CH}_{3}, \mathrm{X}=\mathrm{CH}_{2}, \mathrm{Y}-\mathrm{Z}=\mathrm{CH}_{2} \mathrm{CH}_{2}$

Reagents and conditions: (i) $\mathrm{AcONa} / \mathrm{Ac}_{2} \mathrm{O}$, reflux; (ii) $\mathrm{H}_{2}, 5 \% \mathrm{Pd} / \mathrm{C}, \mathrm{MeOH}$, r.t.; (iii) $\mathrm{SOCl}_{2}$, reflux; (iv) for $\mathrm{GA}, \mathrm{H}_{2} \mathrm{SO}_{4} / \mathrm{MeOH}$; for $\mathrm{OA}$, $\mathrm{EtBr}$, $\mathrm{KI} / \mathrm{K}_{2} \mathrm{CO}_{3}$ /acetone, reflux; (v) pyridine/ $\mathrm{CH}_{2} \mathrm{Cl}_{2}$, r.t.; (vi) $\mathrm{MeONa} / \mathrm{MeOH}$, r.t., and then resin $\mathrm{H}^{+}$form

\section{Scheme 1}

\section{2 合成}

\subsection{1 二乙酰基咖啡酸 $(2)$ 的合成}

将咖啡酸 1 (18.01 g, $0.1 \mathrm{~mol})$ 溶于 $200 \mathrm{~mL}$ 醋酸酐中, 加入无水醋酸钠 $(1.66 \mathrm{~g}, 0.02 \mathrm{~mol})$, 加热回流 $1 \mathrm{~h}$, 冷却 至室温后, 剧烈摚拌下将反应液倾入 $2 \mathrm{~L}$ 冰水中, 充分 搅拌 $3 \mathrm{~h}$. 混合液抽滤, 固体水洗三次, 烘干后得浅黄色 固体 $\mathbf{2}^{[11,12]}(23.50 \mathrm{~g}$, 产率 $89 \%$. 熔点与文献相同).

1.2.2 氢化二乙酰基咖啡酸(3)的合成

将化合物 $2(2.64 \mathrm{~g}, 10 \mathrm{mmol})$ 溶于 $50 \mathrm{~mL}$ 甲醇中, 加 入 $5 \%$. Pd-C 催化剂 $(0.10 \mathrm{~g})$, 在氢气气氛中常温常压摚 拌 $2 \mathrm{~h}$, TLC 检测无原料. 滤去 Pd-C 后滤液脱溶剂得白色 固体 3 (2.50 g, 产率 94\%.). ${ }^{1} \mathrm{H} \mathrm{NMR}\left(300 \mathrm{MHz}, \mathrm{CDCl}_{3}\right)$ $\delta: 2.28\left(\mathrm{~s}, 3 \mathrm{H}, \mathrm{O}=\mathrm{CCH}_{3}\right), 2.29\left(\mathrm{~s}, 3 \mathrm{H}, \mathrm{O}=\mathrm{CCH}_{3}\right), 2.68(\mathrm{t}$, $\left.J=7.8 \mathrm{~Hz}, 2 \mathrm{H}, \mathrm{CH}_{2}\right), 2.96\left(\mathrm{t}, J=7.8 \mathrm{~Hz}, 2 \mathrm{H}, \mathrm{CH}_{2}\right), 7.04$ (s, 1H, ArH), 7.10 (s, 2H, ArH).

\section{2 .3 甘草次酸甲酯(6)的合成}

将甘草次酸 $(4.70 \mathrm{~g}, 10 \mathrm{mmol})$ 溶于 $50 \mathrm{~mL}$ 甲醇中, 加入 $2 \mathrm{~mL}$ 浓硫酸, 加热回流 $3 \mathrm{~h}$. 冷却至室温后, 将反 应液倾入 $150 \mathrm{~mL}$ 水中, 充分搅拌, 用二氯甲烷萃取 (50 $\mathrm{mL} \times 3)$, 合并有机相, 水洗, 无水硫酸钠干燥, 减压脱
溶剂后得白色固体 $\boldsymbol{6}^{[13]} 4.21 \mathrm{~g}$, 产率 87\%. m.p. 256.0 $258.0{ }^{\circ} \mathrm{C}$ (文献值: $254 \sim 258{ }^{\circ} \mathrm{C}$ ).

\subsection{4 齐墩果酸乙酯(7)的合成}

在 $250 \mathrm{~mL}$ 的圆底烧瓶中将齐墩果酸 $(4.56 \mathrm{~g}, 10$ $\mathrm{mmol}$ )溶于干燥的丙酮 $(120 \mathrm{~mL})$ 中, 加入碳酸钾 $(5.53 \mathrm{~g}$, $40 \mathrm{mmol})$ 、碘化钾 $(0.33 \mathrm{~g}, 2 \mathrm{mmol})$ 、溴乙烷 $(4.36 \mathrm{~g}, 40$ $\mathrm{mmol}$ ), 加热回流反应 $4 \mathrm{~h}$. 冷却至室温后, 将反应液倾 入 $200 \mathrm{~mL}$ 水中, 搅拌, 二氯甲烷萃取 $(50 \mathrm{~mL} \times 3)$, 有机 相水洗, 无水硫酸钠干燥, 减压脱溶剂后得到白色固体 $7^{[14]} 4.55 \mathrm{~g}$, 产率 $94 \%$ (熔点与文献相同).

\subsection{5 化合物 8 的合成}

3-O-二乙酰基咖啡酰甘草次酸甲酯(8a): 将化合物 $2(1.6 \mathrm{~g}, 6 \mathrm{mmol})$ 溶于 $20 \mathrm{~mL}$ 二氯亚砜中, 加热回流 $2 \mathrm{~h}$. 减压蒸去剩余的二氯亚砜得到二乙酰基咖啡酰氯 4 粗产 品. 在 $\mathrm{N}_{2}$ 保护下将化合物 4 粗产品溶于 $30 \mathrm{~mL}$ 干燥的二 氯甲烷中, 加入化合物 $6(0.96 \mathrm{~g}, 2 \mathrm{mmol})$ 、吡啶 $(0.5 \mathrm{~g}, 6$ $\mathrm{mmol})$, 室温摚拌反应 $4 \mathrm{~h}$. 反应液浓缩后直接用柱色谱 分离 $[V$ (石油醚) $: V($ 乙酸乙酯 $)=3: 1]$, 得到 $3-O-$ 二乙 酰基咖啡酰甘草次酸甲酯(8a) $1.12 \mathrm{~g}$, 产率 72\%. 浅黄色 固体, m.p. 267.0 268.5 ${ }^{\circ} \mathrm{C} ;{ }^{1} \mathrm{H}$ NMR $\left(300 \mathrm{MHz}, \mathrm{CDCl}_{3}\right)$ $\delta: 7.60(\mathrm{~d}, J=15.9 \mathrm{~Hz}, 1 \mathrm{H},=\mathrm{CH}), 7.40(\mathrm{~d}, J=15.8 \mathrm{~Hz}$, 
2H, ArH), $7.13 \sim 7.31(\mathrm{~m}, 1 \mathrm{H}, \mathrm{ArH}), 6.39(\mathrm{~d}, J=15.9 \mathrm{~Hz}$, $1 \mathrm{H},=\mathrm{CH}), 5.68(\mathrm{~s}, 1 \mathrm{H}, \mathrm{H}-12), 4.65(\mathrm{dd}, J=4.8,11.4 \mathrm{~Hz}$, $1 \mathrm{H}, \mathrm{H}-3), 3.69\left(\mathrm{~s}, 3 \mathrm{H}, \mathrm{O}=\mathrm{COCH}_{3}\right), 2.80 \sim 2.88(\mathrm{~m}, 1 \mathrm{H}$, $\mathrm{H}-18$ ), 2.39 (s, 1H, H-9), 2.31 (s, 3H, O= $\mathrm{CCH}_{3}$ ), 2.30 (s, $\left.3 \mathrm{H}, \mathrm{O}=\mathrm{CCH}_{3}\right), 1.81 \sim 2.06(\mathrm{~m}, 4 \mathrm{H}), 1.57 \sim 1.66(\mathrm{~m}, 6 \mathrm{H})$, $1.29 \sim 1.42(\mathrm{~m}, 9 \mathrm{H}), 1.32(\mathrm{~s}, 3 \mathrm{H}), 1.25(\mathrm{~s}, 3 \mathrm{H}), 1.18(\mathrm{~s}$, 3H), 1.17 (s, 3H), 0.99 (s, 3H), 0.88 (s, 3H), 0.83 (s, 3H); IR (KBr) v: 2935, 2864, 1770, 1723, $1630 \mathrm{~cm}^{-1}$.

3- $O$-二乙酰基咖啡酰齐墩果酸乙酯 $(\mathbf{8 b})$ : 与化合物 $\mathbf{8 a}$ 的合成相同，由化合物 $\mathbf{2}$ 和化合物 7 制得，产率 $75 \%$. m.p. 206.0 208.0 ${ }^{\circ} \mathrm{C}$; ${ }^{1} \mathrm{H}$ NMR (300 $\left.\mathrm{MHz}, \mathrm{CDCl}_{3}\right) \delta$ : $7.59(\mathrm{~d}, J=15.9 \mathrm{~Hz}, 1 \mathrm{H},=\mathrm{CH}), 7.39$ (d, $J=15.8 \mathrm{~Hz}, 2 \mathrm{H}$, ArH), 7.22 (s, 1H, ArH), 6.38 (d, J=15.9 Hz, 1H, =CH), 5.29 (s, 1H, H-12), 4.63 (t, J=7.8 Hz, 1H, H-3), 4.00 $4.20\left(\mathrm{~m}, 2 \mathrm{H}, \mathrm{O}=\mathrm{COCH}_{2}\right), 2.87(\mathrm{dd}, J=4.5,14.4 \mathrm{~Hz}, 1 \mathrm{H}$, $\mathrm{H}-18), 2.31\left(\mathrm{~s}, 3 \mathrm{H}, \mathrm{O}=\mathrm{CCH}_{3}\right), 2.30\left(\mathrm{~s}, 3 \mathrm{H}, \mathrm{O}=\mathrm{CCH}_{3}\right)$, $1.80 \sim 1.88(\mathrm{~m}, 2 \mathrm{H}), 1.51 \sim 1.67(\mathrm{~m}, 10 \mathrm{H}), 1.30 \sim 1.46(\mathrm{~m}$, $2 \mathrm{H}), 1.16 \sim 1.30(\mathrm{~m}, 4 \mathrm{H}), 1.23(\mathrm{t}, J=7.8 \mathrm{~Hz}, 3 \mathrm{H}$, $\left.\mathrm{CH}_{2} \mathrm{CH}_{3}\right), 1.13(\mathrm{~s}, 3 \mathrm{H}), 0.96 \sim 1.10(\mathrm{~m}, 2 \mathrm{H}), 0.92(\mathrm{~s}, 3 \mathrm{H})$, 0.91 (s, 3H), 0.90 (s, 3H), 0.83 (s, 3H), 0.79 (s, 3H), 0.73 (s, 3H); IR (KBr) v: 2935, 2852, 1769, 1728, $1654 \mathrm{~cm}^{-1}$.

3- $O$-氢化二乙酰基咖啡酰甘草次酸甲酯 $(8 \mathrm{c})$ : 与化 合物 $\mathbf{8 a}$ 的合成相同，由化合物 $\mathbf{3}$ 和化合物 $\mathbf{6}$ 制得，产率 68\%. m.p. $186.0 \sim 188.0{ }^{\circ} \mathrm{C} ;{ }^{1} \mathrm{H}$ NMR (300 MHz, $\mathrm{CDCl}_{3}$ ) $\delta: 7.09$ (s, 2H, ArH), 7.04 (s, 1H, ArH), 5.67 (s, 1H, H-12), 4.52 (dd, $J=4.8,11.4 \mathrm{~Hz}, 1 \mathrm{H}, \mathrm{H}-3), 3.69$ (s, 3H, O= $\left.\mathrm{COCH}_{3}\right), 2.95\left(\mathrm{t}, J=7.8 \mathrm{~Hz}, 2 \mathrm{H}, \mathrm{CH}_{2}\right), 2.71 \sim 2.88(\mathrm{~m}, 1 \mathrm{H}$, $\mathrm{H}-18$ ), 2.63 (t, J=7.8 Hz, 2H, $\mathrm{CH}_{2}$ ), 2.35 (s, 1H, H-9), $2.28\left(\mathrm{~s}, 2 \times 3 \mathrm{H}, 2 \times \mathrm{O}=\mathrm{CCH}_{3}\right), 1.81 \sim 2.04(\mathrm{~m}, 4 \mathrm{H})$, $1.58 \sim 1.66(\mathrm{~m}, 6 \mathrm{H}), 1.29 \sim 1.41(\mathrm{~m}, 9 \mathrm{H}), 1.33(\mathrm{~s}, 3 \mathrm{H})$, 1.25 (s, 3H), 1.18 (s, 3H), 1.15 (s, 3H), 0.97 (s, 3H), 0.88 (s, 3H), 0.84 (s, 3H); IR (KBr) v: 2929, 2843, 1778, 1710, $1632 \mathrm{~cm}^{-1}$.

3- $O$-氢化二乙酰基咖啡酰齐墩果酸乙酯 $(\mathbf{8 d})$ : 与化 合物 $\mathbf{8 a}$ 的合成相同，由化合物 $\mathbf{3}$ 和化合物 $\mathbf{7}$ 制得，产率 70\%. m.p. 192.5 194.0 ${ }^{\circ} \mathrm{C} ;{ }^{1} \mathrm{H}$ NMR (300 MHz, $\mathrm{CDCl}_{3}$ ) $\delta: 7.09$ (s, 2H, ArH), 7.04 (s, 1H, ArH), 5.28 (s, 1H, H-12), $4.50(\mathrm{t}, J=7.8 \mathrm{~Hz}, 1 \mathrm{H}, \mathrm{H}-3), 4.02 \sim 4.16(\mathrm{~m}, 2 \mathrm{H}, \mathrm{O}=$ $\left.\mathrm{COCH}_{2}\right), 2.95\left(\mathrm{t}, J=7.8 \mathrm{~Hz}, 2 \mathrm{H}, \mathrm{CH}_{2}\right), 2.86(\mathrm{dd}, J=4.5$, $14.4 \mathrm{~Hz}, 1 \mathrm{H}, \mathrm{H}-18), 2.63$ (t, $\left.J=7.8 \mathrm{~Hz}, 2 \mathrm{H}, \mathrm{CH}_{2}\right), 2.28$ (s, $\left.3 \mathrm{H}, \mathrm{O}=\mathrm{CCH}_{3}\right), 1.74 \sim 1.98(\mathrm{~m}, 2 \mathrm{H}), 1.52 \sim 1.68(\mathrm{~m}, 10 \mathrm{H})$, $1.30 \sim 1.46(\mathrm{~m}, 2 \mathrm{H}), 1.16 \sim 1.32(\mathrm{~m}, 4 \mathrm{H}), 1.22(\mathrm{t}, J=7.8$ $\left.\mathrm{Hz}, 3 \mathrm{H}, \mathrm{CH}_{2} \mathrm{CH}_{3}\right), 1.15$ (s, 3H), $0.94 \sim 1.12(\mathrm{~m}, 2 \mathrm{H}), 0.93$ $(\mathrm{s}, 3 \mathrm{H}), 0.91$ (s, 3H), 0.89 (s, 3H), 0.83 (s, 3H), 0.78 (s, $3 \mathrm{H}), 0.72$ (s, 3H); IR (KBr) v: 2947, 2866, 1776, 1727,
$1636 \mathrm{~cm}^{-1}$.

1.2.6 化合物 9 的合成

将催化量的金属钠溶于甲醇中，加入化合物 $\mathbf{8}$, 室 温搅拌反应 $0.5 \mathrm{~h}, \mathrm{TLC}$ 检测无原料. 加入强酸型阳离子 交换树脂搅拌 $2 \mathrm{~h}$ 后，滤去树脂，滤液脱溶剂，用柱色谱 分离 $[V$ (石油醚)： $V$ (乙酸乙酯 $)=3: 1]$, 即得化合物 9 .

3- $O$-咖啡酰甘草次酸甲酯(9a): 浅黄色固体, 产率 93\%. m.p. 286.0 288.0 ${ }^{\circ} \mathrm{C} ;{ }^{1} \mathrm{H}$ NMR (300 MHz, $\mathrm{CDCl}_{3}$ ) $\delta: 7.59(\mathrm{~d}, J=15.9 \mathrm{~Hz}, 1 \mathrm{H},=\mathrm{CH}), 7.47(\mathrm{~s}, 1 \mathrm{H}, \mathrm{ArH})$, $6.89(\mathrm{~m}, 2 \mathrm{H}, \mathrm{ArH}), 6.25$ (d, $J=15.9 \mathrm{~Hz}, 1 \mathrm{H},=\mathrm{CH}), 5.75$ (s, 1H, H-12), 4.71 (dd, $J=4.5,11.7 \mathrm{~Hz}, 1 \mathrm{H}, \mathrm{H}-3), 3.73$ (s, $\left.3 \mathrm{H}, \mathrm{O}=\mathrm{COCH}_{3}\right), 2.76 \sim 2.89(\mathrm{~m}, 1 \mathrm{H}, \mathrm{H}-18), 2.49(\mathrm{~s}, 1 \mathrm{H}$, H-9), $1.81 \sim 2.03(\mathrm{~m}, 4 \mathrm{H}), 1.59 \sim 1.66(\mathrm{~m}, 6 \mathrm{H}), 1.29 \sim 1.43$ $(\mathrm{m}, 9 \mathrm{H}), 1.35$ (s, 3H), 1.27 (s, 3H), 1.19 (s, 3H), 1.17 (s, $3 \mathrm{H}), 0.98$ (s, 3H), 0.89 (s, 3H), 0.83 (s, 3H); IR (KBr) v: 3542, 3412, 3243, 2951, 2860, 1704, $1634 \mathrm{~cm}^{-1}$; HRMS calcd for $\mathrm{C}_{40} \mathrm{H}_{55} \mathrm{O}_{7}[\mathrm{M}+\mathrm{H}]^{+}$: 647.3948 , found 647.3942 .

3- $O$-咖啡酰齐墩果酸乙酯 $(9 \mathbf{b})$ : 淡黄色固体, 产率 90\%. m.p. 228.0 $230.0{ }^{\circ} \mathrm{C} ;{ }^{1} \mathrm{H}$ NMR (300 $\mathrm{MHz}, \mathrm{CDCl}_{3}$ ) $\delta: 7.57(\mathrm{~d}, J=15.9 \mathrm{~Hz}, 1 \mathrm{H},=\mathrm{CH}), 7.13(\mathrm{~s}, 1 \mathrm{H}, \mathrm{ArH})$, $6.95 \sim 7.06(\mathrm{~m}, 1 \mathrm{H}, \mathrm{ArH}), 6.84 \sim 6.93(\mathrm{~m}, 1 \mathrm{H}, \mathrm{ArH}), 6.27$ $(\mathrm{d}, J=15.9 \mathrm{~Hz}, 1 \mathrm{H},=\mathrm{CH}), 5.27(\mathrm{~s}, 1 \mathrm{H}, \mathrm{H}-12), 4.61(\mathrm{t}$, $J=8.1 \mathrm{~Hz}, 1 \mathrm{H}, \mathrm{H}-3), 4.00 \sim 4.18\left(\mathrm{~m}, 2 \mathrm{H}, \mathrm{O}=\mathrm{COCH}_{2}\right)$, $2.86(\mathrm{dd}, J=4.5,14.4 \mathrm{~Hz}, 1 \mathrm{H}, \mathrm{H}-18), 1.78 \sim 1.94(\mathrm{~m}, 2 \mathrm{H})$, $1.54 \sim 1.69(\mathrm{~m}, 10 \mathrm{H}), 1.30 \sim 1.44(\mathrm{~m}, 2 \mathrm{H}), 1.05 \sim 1.33(\mathrm{~m}$, $4 \mathrm{H}), 1.23\left(\mathrm{t}, J=7.8 \mathrm{~Hz}, 3 \mathrm{H}, \mathrm{CH}_{2} \mathrm{CH}_{3}\right), 1.13(\mathrm{~s}, 3 \mathrm{H}), 0.94 \sim$ $1.20(\mathrm{~m}, 2 \mathrm{H}), 0.93(\mathrm{~s}, 3 \mathrm{H}), 0.91(\mathrm{~s}, 3 \mathrm{H}), 0.90(\mathrm{~s}, 3 \mathrm{H}), 0.84$ (s, 3H), 0.78 (s, 3H), 0.73 (s, 3H);. IR (KBr) v: 3542, 3393, 2947, 2873, 1706, $1632 \mathrm{~cm}^{-1}$; HRMS calcd for $\mathrm{C}_{41} \mathrm{H}_{59} \mathrm{O}_{6}$ $[\mathrm{M}+\mathrm{H}]^{+}: 647.4312$, found 647.4308 .

$3-O$-氢化咖啡酰甘草次酸甲酯 $(9 \mathrm{c})$ : 白色固体，产 率 95\%. m.p. $150.0 \sim 152.0{ }^{\circ} \mathrm{C} ;{ }^{1} \mathrm{H}$ NMR $(300 \mathrm{MHz}$, $\left.\mathrm{CDCl}_{3}\right) \delta: 6.76(\mathrm{~d}, J=15.8 \mathrm{~Hz}, 2 \mathrm{H}, \mathrm{ArH}), 6.63(\mathrm{~s}, 1 \mathrm{H}$, ArH), 5.68 (s, 1H, H-12), 4.51 (dd, $J=4.8,11.2 \mathrm{~Hz}, 1 \mathrm{H}$, $\mathrm{H}-3), 3.69\left(\mathrm{~s}, 3 \mathrm{H}, \mathrm{O}=\mathrm{COCH}_{3}\right), 2.85(\mathrm{t}, J=7.8 \mathrm{~Hz}, 2 \mathrm{H}$, $\left.\mathrm{CH}_{2}\right), 2.70 \sim 2.85(\mathrm{~m}, 1 \mathrm{H}, \mathrm{H}-18), 2.58(\mathrm{t}, J=7.8 \mathrm{~Hz}, 2 \mathrm{H}$, $\left.\mathrm{CH}_{2}\right), 2.37$ (s, 1H, H-9), $1.79 \sim 2.04(\mathrm{~m}, 4 \mathrm{H}), 1.59 \sim 1.67$ $(\mathrm{m}, 6 \mathrm{H}), 1.31 \sim 1.44(\mathrm{~m}, 9 \mathrm{H}), 1.32(\mathrm{~s}, 3 \mathrm{H}), 1.27(\mathrm{~s}, 3 \mathrm{H})$, 1.18 (s, 3H), 1.15 (s, 3H), 0.97 (s, 3H), 0.90 (s, 3H), 0.83 (s, 3H); IR (KBr) v: 3422, 2949, 2871, 1730, $1641 \mathrm{~cm}^{-1}$; HRMS calcd for $\mathrm{C}_{40} \mathrm{H}_{57} \mathrm{O}_{7}[\mathrm{M}+\mathrm{H}]^{+}:$649.4104, found 649.4109.

3- $O$-氢化咖啡酰齐墩果酸乙酯 $(9 \mathrm{~d})$ : 白色固体, 产 率 91\%. m.p. 202.0 203.0 ${ }^{\circ} \mathrm{C}$; ${ }^{1} \mathrm{H}$ NMR $(300 \mathrm{MHz}$, $\left.\mathrm{CDCl}_{3}\right) \delta: 6.61 \sim 6.80(\mathrm{~m}, 3 \mathrm{H}, \mathrm{ArH}), 5.27(\mathrm{~s}, 1 \mathrm{H}, \mathrm{H}-12)$, 
$4.49(\mathrm{t}, J=7.8 \mathrm{~Hz}, 1 \mathrm{H}, \mathrm{H}-3), 4.00 \sim 4.18(\mathrm{~m}, 2 \mathrm{H}, \mathrm{O}=$ $\mathrm{COCH}_{2}$ ), 2.88 (dd, $\left.J=4.5,14.4 \mathrm{~Hz}, 1 \mathrm{H}, \mathrm{H}-18\right), 2.84$ (t, $J=$ $\left.7.8 \mathrm{~Hz}, 2 \mathrm{H}, \mathrm{CH}_{2}\right), 2.58\left(\mathrm{t}, J=7.8 \mathrm{~Hz}, 2 \mathrm{H}, \mathrm{CH}_{2}\right), 1.76 \sim$ $1.92(\mathrm{~m}, 2 \mathrm{H}), 1.50 \sim 1.65(\mathrm{~m}, 10 \mathrm{H}), 1.30 \sim 1.45(\mathrm{~m}, 2 \mathrm{H})$, $1.17 \sim 1.29(\mathrm{~m}, 4 \mathrm{H}), 1.23\left(\mathrm{t}, J=7.8 \mathrm{~Hz}, 3 \mathrm{H}, \mathrm{CH}_{2} \mathrm{CH}_{3}\right)$, $1.12(\mathrm{~s}, 3 \mathrm{H}), 0.97 \sim 1.09(\mathrm{~m}, 2 \mathrm{H}), 0.93(\mathrm{~s}, 3 \mathrm{H}), 0.92(\mathrm{~s}$, $3 \mathrm{H}), 0.91$ (s, 3H), 0.83 (s, 3H), 0.79 (s, 3H), 0.74 (s, 3H); IR (KBr) v: 3557, 3381, 3250, 2944, 2874, 1709, 1662 $\mathrm{cm}^{-1}$; HRMS calcd for $\mathrm{C}_{41} \mathrm{H}_{61} \mathrm{O}_{6}[\mathrm{M}+\mathrm{H}]^{+}:$649.4468, found 649.4471 .

\section{3 保肝活性测试}

采用抗异硫氰酸荎酯所致肝损伤实验测试.

\subsection{1 材料}

健康雄性昆明小鼠 160 只, 体重 $(20 \pm 2) \mathrm{g}$, 由上海 中医药大学附属曙光医院实验动物房提供. 将小鼠按随 机数字表法分为 11 组, 即正常组 10 只、病理模型组 $(15$ 只)、咖啡酸组(15 只)、甘草次酸组(15 只)、齐墩果酸组 (15 只)、咖啡酸十甘草次酸组(15 只)、咖啡酸十齐墩果 酸组 $(15$ 只)、3- $O$-咖啡酸甘草次酸甲酯 $(9 \mathbf{a}, 15$ 只)、3- $O-$ 咖啡酸齐墩果酸乙酯 $(9 \mathbf{b}, 15$ 只)、3- $O-$ 二氢咖啡酸甘草 次酸甲酯组 $(9 \mathbf{c}, 15$ 只)、3- $O$-二氢咖啡酸齐墩果酸乙酯 (9d, 15 只). 所有小鼠于试验前 $12 \mathrm{~h}$ 禁食, 但自由饮水.

咖啡酸、甘草次酸、齐墩果酸市售产品. 3-O-咖啡 酸甘草次酸甲酯 $(9 \mathbf{a}), 3-O$ - 咖啡酸齐墩果酸乙酯 $(9 \mathbf{b})$, 3- $O$-二氢咖啡酸甘草次酸甲酯 $(9 \mathrm{c}), 3-O-$-二氢咖啡酸齐 墩果酸乙酯(9d)自制, 纯度 $>99 \%$ (HPLC).

异硫氧酸萗酯 $(\alpha$-Naphthyl isothiocyanate, ANIT), $\mathrm{CP}$ (化学纯), 北京通县育才精细化工厂, NO 911209; 谷 丙转氨酶( $A L T, I U / L)$ 、谷草转氨酶 $(A S T, I U / L)$ 、直接胆 红素 $(\mathrm{DB}, u m o l / L) 、$ 总胆汁酸 $(\mathrm{BA}, \mathrm{umol} / \mathrm{L}) 、$ 尿素氮 $(B U N$, $\mathrm{mmol} / \mathrm{L})$ 、肌酐 $(\mathrm{CR}, \mathrm{umol} / \mathrm{L})$ 测定试剂盒, 贝克曼库尔特 公司 DXC800 全自动生化分析仪专用试剂盒.
DXC800 全自动生化分析仪(美国贝克曼库尔特公 司).

\subsection{2 方法}

除正常组外, 其它小鼠采用 ANIT 溶于适量植物油 中配制成 $14 \mathrm{mg} / \mathrm{mL}$ 浓度, 在实验第 $1 \mathrm{~d}$ 以 ANIT 70 $\mathrm{mg} / \mathrm{kg}$ 剂量以 $0.1 \mathrm{~mL} / 20 \mathrm{~g}$ 经口灌胃造模, 实验第 $2,3 \mathrm{~d}$ 分别采用咖啡酸, 甘草次酸, 齐墩果酸, 咖啡酸十甘草 次酸, 咖啡酸十齐墩果酸, 3- $O$-咖啡酸甘草次酸甲酯 (9a), 3- $O$-咖啡酸齐墩果酸乙酯 $(9 \mathbf{b}), 3-O-$ 二氢咖啡酸甘 草次酸甲酯 $(\mathbf{9 c}), 3-O-$ 二氢咖啡酸齐墩果酸乙酯 $(\mathbf{9 d})$ 各 $50 \mu \mathrm{mol} / \mathrm{kg}$ 以 $0.1 \mathrm{~mL} / 20 \mathrm{~g}$ 经口给药, 病理对照组采用等 体积蒸馏水经口给药, 未次给药 $24 \mathrm{~h}($ 染毒 $72 \mathrm{~h}$ )后处死 收集样本供检测.

血清肝肾功能指标检测参照试剂盒说明书上机进 行检测. 实验结果见表 1 所示

\section{2 结果与讨论}

\section{1 合成}

在合成齐墩果酸乙酯(7)的过程中, 由于齐墩果酸 28-位羧基空间位阻较大, 采用酸催化与醇直接酯化法 不能实现 ${ }^{[15]}$, 而采用丙酮作溶剂, 碳酸钾作除酸剂, 在 碘化钾催化下用溴乙烷与齐墩果酸反应, 成功地以较高 收率制得化合物 7.

在咖啡酸与萜类酯化合物对接的合成过程中, 由于 萜类酯化合物 6 和 7 的极性与相应的对接产物 8 极为接 近, 因而使柱色谱分离二者变得相当困难. 在实验操作 中, 我们使用过量的酰化试剂, 即化合物 $\mathbf{2}$ 和 $\mathbf{3}$, 促使化 合物 6 和 7 完全反应, 不仅提高了化合物 8 的产率, 也 使分离提纯过程得到简化. 化合物 8 在催化量的甲醇钠 甲醇溶液中,脱去乙酰基后，加入强酸型阳离子交换树 脂, 以中和体系中的碱并除去钠离子, 使目标化合物 9 的分离变得简单, 省去了水洗萃取过程中带来的不必要

表 1 血清肝肾功能指标实验数据 ${ }^{a}$

Table 1 Laboratory index of serum liver and renal functions

\begin{tabular}{lcccccc}
\hline \multicolumn{1}{c}{ 化合物 } & ALT & AST & DB & BA & BUN & CR \\
\hline 咖啡酸 & 1153.36 & 1225.38 & 1248.50 & 204.57 & 49.20 & 28.07 \\
甘草次酸 & 1406.56 & 1053.13 & 1245.78 & 214.06 & 54.17 & 31.86 \\
齐墩果酸 & 1142.81 & 1280.47 & 941.44 & 203.56 & 50.69 & 30.62 \\
咖啡酸十甘草次酸 & 911.41 & 972.53 & 1119.47 & 193.00 & 51.18 & 28.15 \\
咖啡酸十齐墩果酸 & 955.19 & 1097.20 & 967.13 & 196.44 & 60.94 & 33.82 \\
9a & 740.79 & 1074.86 & 653.80 & 224.25 & 60.60 & 38.17 \\
9b & 832.44 & 1133.21 & 878.84 & 196.42 & 57.79 & 30.82 \\
9c & 726.00 & 840.35 & 645.65 & 224.60 & 59.55 & 26.33 \\
9d & 993.27 & 1011.60 & 1091.71 & 357.53 & 57.27 & 29.73 \\
模型 & 926.63 & 1196.50 & 1116.33 & 364.67 & 86.22 & 36.00 \\
正常 & 41.00 & 132.86 & 38.00 & 5.57 & 85.71 & 15.67 \\
\hline
\end{tabular}

${ }^{a} \mathrm{ALT}($ 谷丙转氨酶)、 $\mathrm{AST}($ 谷草转氨酶)、 $\mathrm{DB}$ (直接胆红素)、BA(胆汁酸)均为反应肝细胞损伤的指标, 而 BUN(尿素氮)和 CR(肌酩)则为反应肾功能的指标. 
损失.

\section{2 保肝活性}

血清肝肾功能指标见表 1 . 由表中数据可以看出, 经过化合物 9c 给药的小白鼠各项指标与模型相比均有 明显改善, 可见该化合物具有降酶(谷丙转氨酶, 谷草 转氨酶)和退黄(直接胆红素)的双重效果, 同时对肾功能 也有保护作用.

\section{3 结论}

综上所述，采用侧基保护、酰化与去保护等步骤， 将咖啡酰基及氢化咖啡酰引入到甘草次酸和齐墩果酸 的 3-位羟基上, 以较高收率合成了 4 个天然的咖啡酸、 氢化咖啡酸与甘草次酸和齐墩果酸对接的新化合物, 初 步药物活性测定显示, 化合物 9c 保护肝功能的效果最 好, 有降酶(谷丙转氨酶, 谷草转氨酶)和退黄(直接胆红 素)的双重效果，同时对肾功能也有保护作用.

\section{References}

[1] Finney, R. S. H.; Tarknoy, A. L. J. Pharm. 1996, 12, 49.

[2] Huang, W.; Huang, J.-Q.; Zhang, D.-F.; Liao, Z.-Q. Chin. J. Integrated Trad. Western Med. Liver Diseases 2003, 13(3), 148 (in Chinese).

(黄炜，黄济群，张东方，廖兆全，中西医结合肝病杂志，2003，
13(3), 148.)

[3] Farina, C.; Pinza, M.; Pifferi, G. Farmaco 1998, 53, 22.

[4] Hu, Z.-H. Acta Pharm. Sinica 1998, 23, 553.

[5] Liu, L.-J.; Yong, J.-P.; Wang, J.-W. Chem. J. Chin. Univ. 2006, 27, 1669 (in Chinese).

(刘利平, 雍建平, 王建武, 高等学校化学学报, 2006, 27, 1669.)

[6] Zhong, C.-Y. Trad. Med. 1975, 6, 47.

[7] Gupta, M. B. Eur. J. Pharm. 1969, 6, 67.

[8] Iwai, K.; Kishimoto, N.; Kakino, Y. J. Agric. Food Chem. 2004, 52, 4893.

[9] Chen, B.; Duan, H.; Takaishi, Y. Phytochemistry 1999, 51, 683.

[10] Yang, Z.-G.; Li, H.-R.; Wang, L.-Y.; Li, Y.-H.; Lu, A.-G.; Wen, X.-F.; Wang, J.; Daikonya, A.; Kitanaka, S. Chem. Pharm. Bull. 2007, 55(1), 15.

[11] Haslam, E.; Makinson, G. K.; Naumann, M. O.; Jill, C. J. Chem. Soc. 1964, 2137.

[12] Li, Z.-J.; Huang, H.-Q.; Cai, M.-S. Chemistry 1993, 56(1), 49 (in Chinese).

(李中军, 黄河清, 蔡孟深, 化学通报, 1993, 56(1), 49.)

[13] Liu, W.-C.; Luo, Y.-Q.; Zhang, Y.-L.; Zhang, C.-X. J. Northeast Normal Univ. (Nat. Sci. Ed.) 2007, 39(4), 154 (in Chinese). (刘文丛, 罗云清, 郑友兰, 张崇禧, 东北师大学报 (自然科学 版), 2007, 39(4), 154.)

[14] Chen, J.; Liu, J.; Zhang, L.-Y.; Wu, G.-Z.; Hua, W.-Y.; Wua, X.-M.; Suna, H.-B. Bioorg. Med. Chem. Lett. 2006, 16, 2915.

[15] Chen, L.; Kong, X.-W.; Zhang, Y.-H. Chin. Trad. Herb. Drugs 2003, 34(12), 1080 (in Chinese).

(陈莉, 孔祥文, 张奕华, 中草药, 2003, 34(12), 1080.)

(Lu, Y.; Lu, Z.) 